ANNALES

POLONICI MATHEMATICI

$84.3(2004)$

\title{
Remarks on pluripolar hulls
}

\author{
by Le Mau Hai, Nguyen Quang Dieu and Tang Van Long (Hanoi)
}

Abstract. The aim of the paper is to establish some results on pluripolar hulls and to define pluripolar hulls of certain graphs.

1. Introduction. Let $\Omega$ be a domain in $\mathbb{C}^{n}$. An upper semicontinuous function $u$ on $\Omega$ is called plurisubharmonic if the restriction of $u$ to the intersection of $\Omega$ with every complex line is subharmonic (we allow the function identically $-\infty$ to be plurisubharmonic). The cone of plurisubharmonic functions (resp. negative plurisubharmonic functions) is denoted by $\mathcal{P S H}(\Omega)$ (resp. $\mathcal{P} \mathcal{S H}^{-}(\Omega)$ ). A subset $E$ of $\mathbb{C}^{n}$ is called pluripolar if for every $a \in A$ we can find a neighbourhood $U_{a}$ of $a$ and $u \in \mathcal{P S H}\left(U_{a}\right)$ such that $u \equiv-\infty$ on $E \cap U_{a}$ and $u \not \equiv-\infty$. A basic theorem of Josefson (see [Kl, Theorem 4.7.4]) asserts that if $E$ is pluripolar in $\Omega$ then there exists a plurisubharmonic function $u$ on $\mathbb{C}^{n}$ such that $u \equiv-\infty$ on $E$ but $u \not \equiv-\infty$. If $E$ is pluripolar and contained in some domain $\Omega$ of $\mathbb{C}^{n}$ then we say that $E$ is complete pluripolar in $\Omega$ if there exists $u \in \mathcal{P S H}(\Omega)$ such that $u^{-1}(-\infty)=E$.

It is easy to see that every complete pluripolar set $E \subset \Omega$ is a $G_{\delta}$ set. In the case $\Omega \subset \mathbb{C}$ by Deny's theorem (see e.g. [Lan]) every polar $G_{\delta}$ subset of $\Omega$ is complete polar. However, in higher dimensions the situation is much more complicated: the set $E=\{(z, 0):|z|<1\}$ is closed and complete pluripolar in the bidisk $\Omega=\{(z, w):|z|<1,|w|<1\}$, but is not complete pluripolar in any domain larger than $\bar{\Omega}$.

In order to see more concretely how a pluripolar subset $E$ of $\Omega$ "propagates", following Levenberg and Poletsky we introduce two types of pluripolar hulls of $E$ relative to $\Omega$ :

$$
\begin{aligned}
& E_{\Omega}^{*}=\bigcap\left\{z \in \Omega: u(z)=-\infty, u \in \mathcal{P S H}(\Omega),\left.u\right|_{E} \equiv-\infty\right\}, \\
& E_{\Omega}^{-}=\bigcap\left\{z \in \Omega: u(z)=-\infty, u \in \mathcal{P S H}^{-}(\Omega),\left.u\right|_{E} \equiv-\infty\right\} .
\end{aligned}
$$

2000 Mathematics Subject Classification: Primary 31C10; Secondary 32U10.

Key words and phrases: plurisubharmonic function, pluripolar set, pluripolar hull, complete pluripolar set, holomorphic function. 
Obviously $E=E_{\Omega}^{*}$ if $E$ is complete pluripolar. On the other hand, $E=E_{\Omega}^{*}$ does not imply $E$ is complete pluripolar. Indeed, every countable and non$G_{\delta}$ set $E$ in $\mathbb{C}$ satisfies $E_{\mathbb{C}}^{*}=E$ but of course $E$ is not complete polar (in $\mathbb{C}$ ). Nevertheless, this implication is true if we assume in addition that $\Omega$ is pseudoconvex and $E$ is both $F_{\sigma}$ and $G_{\delta}$ (see Proposition 2.1 in [Ze]). One of our aims is to discuss variants of the above mentioned result of Zeriahi where the emphasis is on regularity of plurisubharmonic functions whose singular locus coincides with $E$. In particular, we show in Theorem 3.2 that if $E$ is closed in $\Omega$ and $E_{\Omega^{\prime}}^{*} \cap \bar{\Omega}=E$, where $\Omega^{\prime}$ is some domain larger than $\bar{\Omega}$, then there exists a plurisubharmonic function $u$ on $\Omega$, continuous on $\bar{\Omega}$ and strictly plurisubharmonic on $\Omega \backslash E$ such that $u^{-1}(-\infty)=E$.

In general, it is quite difficult to determine $E_{\Omega}^{*}$ and $E_{\Omega}^{-}$; even simple looking sets like $F_{1}=\left\{\left(z, z^{\alpha}\right): z \neq 0\right\}, \alpha>0, \alpha \notin \mathbb{Q}$, or $F_{2}=$ $\left\{\left(z, e^{-1 / z}\right): z \neq 0\right\}$ require considerable efforts (see [LP], [Wi1]) to establish their pluripolar hulls. Combining the description of $\left(F_{2}\right)_{\mathbb{C}^{2}}^{*}$ with Zeriahi's theorem, one can even show that $F_{2}$ is complete pluripolar in $\mathbb{C}^{2}$ (for details see [Wi1]). On the other hand, $F_{1}$ is not so, being a non- $G_{\delta}$ set (see [Wi3] for details).

In the interesting paper [LP], a number of useful techniques to study pluripolar hulls have been established by Levenberg and Poletsky. The next goal of the present work is to apply the methods of Levenberg and Poletsky to describe $E_{\Omega}^{*}$ in case $E$ is the graph of a holomorphic function over some pseudoconvex domain $D$ minus a complex hypersurface and $\Omega=D \times \mathbb{C}$. It should be remarked that in one dimension a complete answer has been obtained in the work of Wiegerinck ([Wi2]). Here we encounter technical difficulties as a complex hypersurface in $\mathbb{C}^{n}, n \geq 2$, contains no isolated points. Therefore a complete description of the pluripolar hull in our case is still missing. We are able to obtain a partial answer in Proposition 4.1. Using this result and the above mentioned theorem of Wiegerinck it is not hard to show that $\left(F_{2}^{\prime}\right)_{\mathbb{C}^{3}}^{*}=F_{2}^{\prime} \cup\{(0,0) \times \mathbb{C}\}$, where $F_{2}^{\prime}=\left\{\left(z, w, e^{z / w}\right): w \neq 0\right\}$. In particular, $F_{2}^{\prime}$ is not complete pluripolar.

The next section deals with a new kind of pluripolar hulls taken in the subclass $\mathcal{L}\left(\mathbb{C}^{n}\right)$ of plurisubharmonic functions with logarithmic growth on $\mathbb{C}^{n}$. The main result of this section is that the new pluripolar hull $\widetilde{E}_{\mathbb{C}^{n}}$ coincides with the former, $E_{\mathbb{C}^{n}}^{*}$. This result is inspired by a well known theorem of Siciak stating that every pluripolar set in $\mathbb{C}^{n}$ is the singular locus of a function in $\mathcal{L}\left(\mathbb{C}^{n}\right)$ (see $\left.[\mathrm{Sic}]\right)$.

Acknowledgments. This work is supported by the National Research Program in Natural Sciences. The authors are grateful to Professor Nguyen Van Khue for proposing problems and useful conversations. 
2. Preliminaries. The result below is particularly useful when we want to "localize" $E_{\Omega}^{*}$.

TheOREM 2.1 ([LP]). Let $\Omega$ be a pseudoconvex domain in $\mathbb{C}^{n}$ and $\left\{\Omega_{\nu}\right\}_{\nu=1}^{\infty}$ an increasing sequence of relatively compact domains with $\bigcup_{\nu=1}^{\infty} \Omega_{\nu}=\Omega$. Let $E \subset \Omega$ be a pluripolar set. Then

$$
E_{\Omega}^{*}=\bigcup_{\nu=1}^{\infty}\left(E \cap \Omega_{\nu}\right)_{\Omega_{\nu}}^{-} .
$$

An important tool in the study of pluripolar hulls is the concept of the pluriharmonic measure (see [LP] for initial use of it and [Wi1], [Wi2], [E], [EW], etc. for further developments). Let $\Omega \subset \mathbb{C}^{n}$ be a domain and $E \subset \Omega$. The pluriharmonic measure at $z \in \Omega$ of $E$ relative to $\Omega$ is the number

$$
\omega(z, E, \Omega)=-\sup \left\{u(z): u \in \mathcal{P} \mathcal{S H}(\Omega),\left.u\right|_{E} \leq-1,\left.u\right|_{\Omega} \leq 0\right\} .
$$

The following result provides a connection between the pluriharmonic measure and the pluripolar hull $E_{\Omega}^{-}$.

Lemma $2.2([\mathrm{LP}])$. Let $\Omega$ be a bounded hyperconvex domain in $\mathbb{C}^{n}$, and $E$ be a pluripolar subset of $\Omega$. Then

$$
E_{\Omega}^{-}=\{z \in \Omega: \omega(z, E, \Omega)>0\} .
$$

We also need

Lemma 2.3 ([Wi1]). Let $\Omega$ be a domain in $\mathbb{C}^{n}, E \subset \Omega$ and let $A \subset \Omega \backslash E$ be closed and pluripolar. Then for all $z \in \Omega \backslash A$ we have

$$
\omega(z, E, \Omega)=\omega(z, E, \Omega \backslash A) .
$$

The following result (Proposition 2.1 in [Ze]) characterizes complete pluripolar sets in terms of their pluripolar hulls.

TheOrem 2.4 ([Ze]). Let $\Omega$ be a pseudoconvex domain in $\mathbb{C}^{n}$ and $E \subset \Omega$ an $F_{\sigma}$ set. Then $E$ is a complete pluripolar set in $\Omega$ if and only if $E$ is a $G_{\delta}$ set and $E_{\Omega}^{*}=E$.

Now we turn to the pluripolar hull of a pluripolar set taken in the class $\mathcal{L}$ of plurisubharmonic functions on $\mathbb{C}^{n}$ with logarithmic growth. For a pluripolar subset $E$ of $\mathbb{C}^{n}$ we set

$$
\widetilde{E}_{\mathbb{C}^{n}}=\left\{z: u(z)=-\infty,\left.u\right|_{E} \equiv-\infty, u \in \mathcal{L}\left(\mathbb{C}^{n}\right)\right\} .
$$

It is proved in $[\mathrm{Sic}]$ that for every pluripolar subset $E$ of $\mathbb{C}^{n}$ we can find $u \in \mathcal{L}\left(\mathbb{C}^{n}\right)$ such that $u \not \equiv-\infty$ and $E \subset u^{-1}(-\infty)$. The following theorem of Bedford and Taylor (Theorem 7.2 in $[\mathrm{BT}]$ ) improves this result.

THEOREM 2.5. Let $E$ be a complete pluripolar subset of $\mathbb{C}^{n}$. Then we can find $u \in \mathcal{L}\left(\mathbb{C}^{n}\right)$ such that $u \not \equiv-\infty$ and $u^{-1}(-\infty)=E$. 
Notation. If $\Omega$ is an open subset of $\mathbb{C}^{n}$ then by $\mathcal{H}(\Omega)$ we mean the set of holomorphic functions on $\Omega$. We also denote by $\mathcal{P} \mathcal{S H}^{c}(\Omega)$ the cone of functions $u$ such that $e^{u}$ is continuous on $\bar{\Omega}$ and $u \in \mathcal{P} \mathcal{S H}(\Omega)$.

3. Variations on Zeriahi's theorem. The next result is a minor improvement of Theorem 2.4.

Proposition 3.1. Let $\Omega, \Omega^{\prime}$ be domains in $\mathbb{C}^{n}$ such that $\Omega \subset \Omega^{\prime}$ and $\Omega^{\prime}$ is pseudoconvex. Assume that $E$ is a pluripolar subset of $\Omega$ which is $F_{\sigma}$ and $G_{\delta}$. If

$$
E=E_{\Omega^{\prime}}^{*} \cap \Omega
$$

then there exists a function $u \in \mathcal{P S} \mathcal{H}\left(\Omega^{\prime}\right)$ such that

(a) $u^{-1}(-\infty) \cap \Omega=E$.

(b) $u$ is continuous on $\Omega \backslash E$.

We could say that this result follows from the proof of Zeriahi's theorem. However, for the sake of completeness we indicate the details.

Proof. Since $E$ is $F_{\sigma}$ and $G_{\delta}$ we can express $E$ and $\Omega \backslash E$ as increasing unions of compact subsets:

$$
E=\bigcup_{j \geq 1} K_{j}, \quad \Omega \backslash E=\bigcup_{j} L_{j} .
$$

We also write $\Omega^{\prime}=\bigcup_{j} \Omega_{j}^{\prime}$, where $\Omega_{j}^{\prime}$ are relatively compact subsets of $\Omega^{\prime}$ and satisfy $K_{j} \cup L_{j} \subset \Omega_{j}^{\prime}$ for all $j \geq 1$. Fix $j \geq 1$. Let $a$ be a point in $L_{j}$. Then from (1) we have $a \notin E_{\Omega^{\prime}}^{*}$. Hence, there exists $u_{a}^{(j)} \in \mathcal{P} \mathcal{S H}\left(\Omega^{\prime}\right)$ such that $\left.u_{a}^{(j)}\right|_{E} \equiv-\infty$ and $u_{a}^{(j)}(a)>-\infty$. By composing with a suitable increasing convex function we may assume that

$$
\left.u_{a}^{(j)}\right|_{E} \equiv-\infty, \quad u_{a}^{(j)}(a)>-2 / 3,\left.\quad u_{a}^{(j)}\right|_{\Omega_{j}^{\prime}} \leq-1 / 2 .
$$

Since $\Omega^{\prime}$ is pseudoconvex, using a result of Fornæss and Narasimhan in [FN] we get a sequence $\left\{u_{k}^{(j)}\right\}$ of real-valued, continuous plurisubharmonic functions on $\Omega^{\prime}$ that decrease pointwise to $u_{a}^{(j)}$ on $\Omega^{\prime}$. Applying Dini's theorem we find $k_{a}$ (sufficiently large) such that

$$
\left.u_{k_{a}}^{(j)}\right|_{K_{j}} \leq-2^{j}, \quad u_{k_{a}}^{(j)}(a)>-1,\left.\quad u_{k_{a}}^{(j)}\right|_{\bar{\Omega}_{j}^{\prime}} \leq 0 .
$$

As $u_{k_{a}}^{(j)}$ is continuous there exists a neighbourhood $U_{a}$ of $a$ such that $u_{k_{a}}^{(j)}>$ -1 on $U_{a}$. Now a standard argument using the compactness of $L_{j}$ implies that there exists a continuous plurisubharmonic function $v_{j}$ on $\Omega^{\prime}$ such that

(i) $\left.v_{j}\right|_{K_{j}} \leq-2^{j}$.

(ii) $\left.v_{j}\right|_{L_{j}}>-1$.

(iii) $\left.v_{j}\right|_{\bar{\Omega}_{j}^{\prime}} \leq 0$. 
Then in view of (iii) the series

$$
u(z):=\sum_{j \geq 1} 2^{-j} v_{j}(z)
$$

defines a plurisubharmonic function on $\Omega^{\prime}$. It follows from (i) that $u \equiv-\infty$ on $E$. Furthermore from (ii) we have $u>-\infty$ on $\Omega^{\prime} \backslash E$. Thus (a) is proved. Finally, (b) follows since the series converges uniformly on $L_{j}$ for each $j$ in view of (ii).

It is reasonable to ask if the function $u$ above can be chosen to be continuous on $\Omega$ if $E$ is closed in $\Omega$. We have the following

TheOrem 3.2. Let $\Omega$ be a bounded domain in $\mathbb{C}^{n}$ and $E$ be a closed pluripolar subset of $\Omega$. Assume that

$$
E_{\Omega^{\prime}}^{*} \cap \bar{\Omega}=E,
$$

where $\Omega^{\prime}$ is some domain in $\mathbb{C}^{n}$ that contains $\bar{\Omega}$. Then there exists a function $u \in \mathcal{P S H}^{c}(\Omega)$ such that

(a) $u^{-1}(-\infty) \cap \bar{\Omega}=E$.

(b) $u$ is smooth and strictly plurisubharmonic on $\Omega \backslash E$.

Proof. We divide the proof into two steps.

STEP 1. Let $\Omega^{\prime \prime}$ be a domain satisfying $\Omega \subset \subset \Omega^{\prime \prime} \subset \subset \Omega^{\prime}$. We will show that there exists $v \in \mathcal{P} \mathcal{S H}\left(\Omega^{\prime \prime}\right)$ such that $v<0$ on $\Omega^{\prime \prime}$ and $v^{-1}(-\infty) \cap \bar{\Omega}=E$. Since the proof below is very similar to that of Proposition 3.1, we only sketch it (see also Lemma 4.2 in [EW] for a similar situation). Express $E$ and $\bar{\Omega} \backslash E$ as increasing unions of compact sets:

$$
E=\bigcup_{j \geq 1} K_{j}, \quad \bar{\Omega} \backslash E=\bigcup_{j \geq 1} L_{j} .
$$

Fix $j \geq 1$ and let $a \in L_{j}$. From (2) we get $u_{a} \in \mathcal{P} \mathcal{S H}\left(\Omega^{\prime}\right)$ such that $\left.u_{a}\right|_{E} \equiv-\infty, u_{a}(a)=-2 / 3$, and $\left.u_{a}\right|_{\Omega^{\prime \prime}} \leq-1 / 2$. By taking convolution with standard smooth kernels we get a sequence of real-valued continuous plurisubharmonic functions $\left\{u_{j}\right\}$ on $\Omega^{\prime \prime}$ that decrease pointwise to $u_{a}$ on $\Omega^{\prime \prime}$. Now the rest of the proof goes exactly as that of Proposition 3.1, and hence we omit the details.

STEP 2. Let $\Omega^{\prime \prime}$ and $v$ be as in Step 1 . We will construct a function $u$ satisfying (a) and (b). To this end, we use methods given in Lemma 1.2 of [Sib]. Let $h$ be an increasing convex function on $[0,1]$ such that

$$
h(x)=0 \quad \forall x \in[0,1 / 2], \quad h(x)<1 \quad \forall x \neq 1, \quad h(1)=1 .
$$

Set $\widetilde{v}_{k}=h\left(e^{v / k}\right)$. Clearly $\widetilde{v}_{k} \in \mathcal{P} \mathcal{S H}\left(\Omega^{\prime \prime}\right), \widetilde{v}_{k}$ vanishes on neighbourhoods of $E$ in $\Omega^{\prime \prime}, 0 \leq \widetilde{v}_{k} \leq 1$ and $\lim _{k \rightarrow \infty} \widetilde{v}_{k}=1$ on $\bar{\Omega} \backslash E$. By taking convolution 
with standard smooth kernels and shrinking $\Omega^{\prime \prime}$ we can assume in addition that $\widetilde{v}_{k}$ is continuous on $\Omega^{\prime \prime}$. Set

$$
w_{k}=\max \left\{\widetilde{v}_{1}, \ldots, \widetilde{v}_{k}\right\} .
$$

Then $\left\{w_{k}\right\}$ is an increasing sequence of continuous plurisubharmonic functions on $\Omega^{\prime \prime}$ that vanish on neighbourhoods of $E$ in $\Omega^{\prime \prime}$. Thus by Dini's theorem it converges uniformly on compact subsets of $\Omega \backslash E$. We claim that there exists a sequence $\left\{F_{k}\right\}$ of compact subsets and a sequence $\left\{n_{k}\right\}$ such that

(i) $\operatorname{Int}\left(F_{k-1}\right) \subset F_{k}, \bigcup F_{k}=\bar{\Omega} \backslash E$.

(ii) $\left\{z \in \Omega: \sum_{j=1}^{k-1} w_{n_{j}} \geq 1 / 2\right\} \subset F_{k}$.

(iii) $1-1 / 2^{k} \leq w_{n_{k}}$ on $F_{k}$.

First we choose $n_{1}$ so large that $F_{1}:=\left\{z: w_{n_{1}} \geq 1 / 2\right\} \neq \emptyset$. Assume that $F_{1}, \ldots, F_{k-1}$ and $n_{1}, \ldots, n_{k-1}$ have been chosen. Let $F_{k}$ be any compact set in $\Omega \backslash E$ that contains $\left\{z \in \bar{\Omega}: \sum_{j=1}^{k-1} w_{n_{j}} \geq 1 / 2\right\} \cup \operatorname{Int}\left(F_{k-1}\right)$. This is possible since the latter set is compact and disjoint from $E$. As the sequence $\left\{w_{k}\right\}$ converges to 1 uniformly on $F_{k}$ we can choose $n_{k}$ so large that

$$
w_{n_{k}} \geq 1-1 / 2^{k} \quad \text { on } F_{k} \text {. }
$$

It is clear that the compact sets $F_{k}$ can be taken so that $\bigcup F_{k}=\bar{\Omega} \backslash E$. Thus the claim is valid.

Now we form the series

$$
\widetilde{w}(z)=|z|^{2}+\sum_{k \geq 1}\left(w_{n_{k}}-1\right) .
$$

It defines a plurisubharmonic function on $\Omega$. Moreover from (iii) we deduce that it converges uniformly on $F_{k}$ for every $k \geq 1$. Thus by (i), $\widetilde{w}$ is realvalued, continuous on $\bar{\Omega} \backslash E$. Next (ii) implies that $\widetilde{w} \equiv-\infty$ on $E$ and $\widetilde{w}$ is continuous at every point of $E$. Clearly $\widetilde{w}$ is strictly plurisubharmonic on $\Omega \backslash E$. Let $\varphi$ be a continuous function on $\bar{\Omega}$ such that $\varphi>0$ on $\Omega$ and $\varphi \equiv 0$ on $\partial \Omega$. Now using Richberg's regularization lemma ([Ri]) we get a smooth strictly plurisubharmonic function $u$ on $\Omega \backslash E$ such that $\widetilde{w} \leq u \leq$ $\widetilde{w}+\varphi$. Since $E$ is closed and pluripolar, $u$ can be extended through $E$ to a plurisubharmonic function on $\Omega$ (still denoted by $u$ ). Finally, it is easy to see that $u$ can be extended to a continuous function on $\bar{\Omega}$ and satisfies $u^{-1}(-\infty) \cap \bar{\Omega}=E$.

4. Pluripolar hulls of certain graphs. The next result is an analogue of Proposition 5 in [Wi2]. Needless to say, we rely heavily on Wiegerinck's methods.

Proposition 4.1. Let $D$ be a pseudoconvex domain in $\mathbb{C}^{n}$, and $f \in$ $\mathcal{H}(D \backslash A)$, where $A=\{g=0\}$ and $g$ is a holomorphic function on $D$. 
Denote by $E$ the graph of $f$ in $(D \backslash A) \times \mathbb{C}$. Then

$$
Z=E \cup(A \times \mathbb{C})
$$

is complete pluripolar in $D \times \mathbb{C}$.

Proof. We split the proof into two steps.

SteP 1. We assume that $D$ is bounded, $g$ is holomorphic on a Stein neighbourhood $\widetilde{D}$ of $\bar{D}$ and $f \in \mathcal{H}(\bar{D} \backslash A)$. Let $B$ be a disk around $0 \in \mathbb{C}$. Let $E^{\prime}$ be the graph of $f$ in $(D \backslash A) \times B$ and

$$
Z^{\prime}=E^{\prime} \cup(A \times B) .
$$

We will show that there exists $u \in \mathcal{P} \mathcal{S H}^{-}(D \times B)$ such that $u$ is $-\infty$ exactly on $Z^{\prime}$. By Theorem 1 in [Ch] we can expand

$$
f(z)=\sum_{j \geq 0} \frac{f_{j}(z)}{g^{j}(z)}, \quad z \in D \backslash A,
$$

where $f_{j}$ are holomorphic functions on $\widetilde{D}$ and satisfy

Fix $\delta>0$ so small that

$$
\lim _{j \rightarrow \infty}\left\|f_{j}\right\|_{\bar{D}}^{1 / j}=0 .
$$

$$
K=\{z \in D: d(z, \partial D) \geq \delta,|g(z)| \geq \delta\} \neq \emptyset .
$$

Set

$$
\varepsilon_{j}=\sup _{k \geq j}\left\|f_{k}\right\|_{\bar{D}}^{1 / k}, \quad h_{N}(z, w)=\frac{1}{N} \log \left(\left|\left(w-\sum_{j=0}^{N} \frac{f_{j}(z)}{g^{j}(z)}\right) g^{N}(z)\right|\right),
$$

where the integer $N$ will be chosen later. It is clear that $\varepsilon_{j} \downarrow 0$. Let $M=$ $\sup _{\bar{D}}|g|$. We now make some estimates. On $(K \times B) \cap E$ we have

$$
\begin{aligned}
h_{N}(z, f(z))= & \frac{1}{N} \log \left|\sum_{j \geq N+1} \frac{f_{j}(z)}{g^{j}(z)}\right|+\log |g(z)| \\
\leq & \frac{1}{N} \log \sum_{j \geq N+1}\left|\frac{f_{j}(z)}{g^{j}(z)}\right|+\log |g(z)| \\
\leq & \frac{1}{N} \log \sum_{j \geq N+1}\left|\frac{\varepsilon_{N}^{j}}{g^{j}(z)}\right|+\log |g(z)| \\
\leq & \frac{1}{N} \log \left|\left(\frac{\varepsilon_{N}}{g(z)}\right)^{N+1} \frac{1}{1-\varepsilon_{N} / \delta}\right|+\log |g(z)| \\
= & \left(1+\frac{1}{N}\right)\left(\log \varepsilon_{N}-\log |g(z)|\right)-\frac{1}{N} \log \left|1-\frac{\varepsilon_{N}}{\delta}\right| \\
& +\log |g(z)| \\
\leq & \log \varepsilon_{N}+C_{1},
\end{aligned}
$$


where $C_{1}$ depends on $\delta$ but not on $\varepsilon_{N}$, and $N$ is chosen such that $\varepsilon_{N}<\delta / 2$. Next, let $r>0$. For $z \in K$ and $|w-f(z)|>r$ we have

$$
\begin{aligned}
h_{N}(z, w) & =\frac{1}{N} \log \left|(w-f(z))+\sum_{j \geq N+1} \frac{f_{j}(z)}{g(z)^{j}}\right|+\log |g(z)| \\
& \geq \frac{1}{N} \log || w-f(z)\left|-\sum_{j \geq N+1}\right| \frac{f_{j}(z)}{g(z)^{j}}||+\log \delta \\
& \geq \frac{1}{N} \log \left(r-2\left(\frac{\varepsilon_{N}}{\delta}\right)^{N+1}\right)+\log \delta \geq-C_{2},
\end{aligned}
$$

where $C_{2}>0$ if $N$ is sufficiently large. The last estimate is that, for $C_{3}>0$ large enough,

$$
h_{N}(z, w)<C_{3} \quad \text { on } D \times B,
$$

where $C_{3}$ depends on $M$ and radius of $B$. Next we set

$$
u_{N}=\max \left(h_{N}-C_{3}, \log \varepsilon_{N}\right) .
$$

Choose a sequence $\left\{N_{i}\right\}$ of positive integers and a sequence $\left\{d_{i}\right\}$ of positive numbers with the following properties: $\sum d_{i}<\infty$ but $\sum d_{i} \log \varepsilon_{N_{i}}=-\infty$. This is possible since $\varepsilon_{N} \downarrow 0$. We form the series

$$
u(z, w)=\sum_{i \geq 1} d_{i} u_{N_{i}}(z, w) .
$$

Notice that on $D \times B, u$ is the limit of a decreasing sequence of plurisubharmonic functions. We use (3) to see that $u \equiv-\infty$ on $E \cap(K \times B)$ and hence on $E$. Next from (4) we obtain $u \neq-\infty$ if $w \neq f(z)$. Combining these facts, we conclude that $u$ is plurisubharmonic on $D \times B$, real-valued, continuous away from $E \cup(A \times \mathbb{C})$ and satisfies

$$
E \subset\{(z, w): u(z, w)=-\infty\} \subset E \cup(A \times \mathbb{C}) .
$$

Thus the function $u(z, w)+\log |g|-\log M$ satisfies our conditions.

SteP 2. We show that $Z$ is complete pluripolar in $D \times \mathbb{C}$. Let $\left\{D_{j}\right\}$ be an increasing sequence of relatively compact hyperconvex subdomains of $D$ such that $D=\bigcup D_{j}$. Let $K$ be a closed ball such that $K \subset D_{1}$ and $K \cap A=\emptyset$. Denote by $E_{K}$ the graph of $f$ over $K$. Let $\left\{B_{j}\right\}$ be an increasing sequence of open disks such that $f(K) \subset B_{1}$ and $\mathbb{C}=\bigcup B_{j}$. Then we have

$$
\left(E_{K}\right)_{D_{j} \times B_{j}}^{-}=\left(E \cap\left(D_{j} \times B_{j}\right)\right)_{D_{j} \times B_{j}}^{-} .
$$

On the other hand, by Step 1 we can find a plurisubharmonic function $u_{j}$ on $D_{j} \times B_{j}$ so that $u_{j}=-\infty$ precisely on $\left(D_{j} \times B_{j}\right) \cap Z$. This implies that

$$
\omega\left((z, w), E_{K}, D_{j} \times B_{j}\right)=0 \quad \text { for }(z, w) \in\left(D_{j} \times B_{j}\right) \backslash Z \text {. }
$$


Thus using Lemma 2.2 we find

$$
\left(E_{K}\right)_{D_{j} \times B_{j}}^{-} \subset\left(D_{j} \times B_{j}\right) \cap Z .
$$

Application of this and Theorem 2.1 gives

$$
E_{D \times \mathbb{C}}^{*} \subset(D \times \mathbb{C}) \cap Z .
$$

Let $a \in D \times \mathbb{C} \backslash Z$. Then we can find $v \in \mathcal{P S H}(D \times \mathbb{C})$ such that $v(a) \neq-\infty$ and $v \equiv-\infty$ on $E$. Hence the plurisubharmonic function $\widetilde{v}=v+\log |g|$ is identically $-\infty$ on $Z$, whereas $\widetilde{v}(a) \neq-\infty$. It follows that $Z_{D \times \mathbb{C}}^{*}=Z$. Finally, it is clear that $Z$ is a $G_{\delta}$ as well as an $F_{\sigma}$, and we infer from Theorem 2.4 that $Z$ is complete pluripolar in $D \times \mathbb{C}$.

We now apply this result to study pluripolar hulls of certain graphs which are analogues of [Wi1] (see Theorem 7 in [Wi1]).

Proposition 4.2. Let $D$ be a pseudoconvex domain in $\mathbb{C}^{n}(n \geq 2)$, $p$ and $q$ be holomorphic functions on $D$ such that $q \not \equiv 0, A=\{z \in D: q(z)=0\}$ $\neq \emptyset$ and $A^{\prime}=\{z \in D: p(z)=q(z)=0\}$ is of (complex) codimension 2 in D. Let $\varphi$ be a holomorphic function on $\mathbb{C}$ which is not a polynomial. Denote by $E$ the graph of $f=\varphi(p / q)$ over $D \backslash A$. Then

$$
E_{D \times \mathbb{C}}^{*}=E \cup\left(A^{\prime} \times \mathbb{C}\right) .
$$

In particular, $E$ is not complete pluripolar in $D \times \mathbb{C}$ if $A^{\prime} \neq \emptyset$.

Proof. First we show that $A^{\prime} \times \mathbb{C} \subset E_{D \times \mathbb{C}}^{*}$. We may assume that $A^{\prime} \neq \emptyset$. Fix $z_{0} \in A^{\prime}$ and $c \in \mathbb{C} \backslash\{0\}$. Then $z_{0} \in X_{c}=\{z \in D: p(z)-c q(z)=0\}$. Let $u \in \mathcal{P} \mathcal{S H}(D \times \mathbb{C})$ be such that $u \equiv-\infty$ on $E$. Let $v(z)=u(z, \varphi(c))$. Clearly $v \equiv-\infty$ on $X_{c} \backslash A^{\prime}$. As $A^{\prime}$ is of codimension 2 in $D$ we deduce that $v \equiv-\infty$ on $X_{c}$. In particular $v \equiv-\infty$ on $A^{\prime}$. It follows that $u \equiv-\infty$ on $A^{\prime} \times \mathbb{C}$. Thus the right hand side of (5) is contained in the left hand side. It remains to prove the reverse inclusion. For this, take an arbitrary point $\left(z_{0}, w_{0}\right) \in A \times \mathbb{C}$ such that $p\left(z_{0}\right) \neq 0$; we claim that $\left(z_{0}, w_{0}\right) \notin E_{D \times \mathbb{C}}^{*}$. Indeed, set

$$
F=\{(\xi, \varphi(1 / \xi)): \xi \in \mathbb{C} \backslash\{0\}\}, \quad B=\{z \in D: p(z)=0\} .
$$

As $\varphi$ is not a polynomial, the point $\xi=0$ is an essential singularity for $\varphi(1 / \xi)$. It follows from Theorem 2 in [Wi2] that $F$ is complete pluripolar in $\mathbb{C}^{2}$. By Theorem 2.5 we get a function $u \in \mathcal{L}\left(\mathbb{C}^{2}\right)$ such that $u=-\infty$ precisely on $F$. Define

$$
\widetilde{u}(z, w)=u\left(\frac{q(z)}{p(z)}, w\right)+\log |p(z)| .
$$

Obviously $\widetilde{u} \in \mathcal{P} \mathcal{S H}((D \backslash B) \times \mathbb{C})$. Since $u \in \mathcal{L}\left(\mathbb{C}^{2}\right)$ the function $\widetilde{u}$ is locally bounded from above near every point of $B \times \mathbb{C}$. So it extends through $B \times \mathbb{C}$ to a plurisubharmonic function (still denoted by $\widetilde{u}$ ) on $D \times \mathbb{C}$. As $\widetilde{u} \equiv-\infty$ 
on $E \backslash(B \times \mathbb{C})$, an open subset of $E$, we infer $\widetilde{u} \equiv-\infty$ on $E$. Now the claim follows since $\widetilde{u}\left(z_{0}, w_{0}\right)=u\left(0, w_{0}\right)+\log \left|p\left(z_{0}\right)\right|>-\infty$. On the other hand, using Proposition 4.1 one gets

$$
E_{D \times \mathbb{C}}^{*} \subset E \cup(A \times \mathbb{C}) .
$$

Putting all this together we are done.

5. Hulls in the class $\mathcal{L}\left(\mathbb{C}^{n}\right)$. We start with the following

Proposition 5.1. Let E be a pluripolar subset of $\mathbb{C}^{n}$. Then $E_{\mathbb{C}^{n}}^{*}=\widetilde{E}_{\mathbb{C}^{n}}$.

For simplicity of notation, throughout this section we write $E^{*}=E_{\mathbb{C}^{n}}^{*}$ and $\widetilde{E}=\widetilde{E}_{\mathbb{C}^{n}}$.

Proof of Proposition 5.1. Obviously, $E^{*} \subset \widetilde{E}$. The reverse inclusion is an easy consequence of the Bedford-Taylor theorem (Theorem 2.5). Indeed, let $z_{0} \notin E^{*}$. Then there is $u \in \mathcal{P S H}\left(\mathbb{C}^{n}\right)$ such that $u\left(z_{0}\right)>-\infty$ and $\left.u\right|_{E} \equiv-\infty$. Let $E^{\prime}=\{z: u(z)=-\infty\}$. Then $E^{\prime}$ is complete pluripolar in $\mathbb{C}^{n}$. So using Theorem 2.5, we find $v \in \mathcal{L}\left(\mathbb{C}^{n}\right)$ such that $v$ is $-\infty$ exactly on $E^{\prime}$. It follows that $z_{0} \notin \widetilde{E}$. We are done.

Proposition 5.2. Let $\Omega$ be a domain in $\mathbb{C}^{n}$ and $E$ be a pluripolar subset of $\Omega$. Assume that $E^{*} \cap \Omega=E$ and $E$ is $F_{\sigma}$ and $G_{\delta}$. Then there exists $u \in \mathcal{L}\left(\mathbb{C}^{n}\right)$ such that $u^{-1}(-\infty) \cap \Omega=E$.

Proof. Applying Proposition 3.1 with $\Omega^{\prime}=\mathbb{C}^{n}$ we obtain $v \in \mathcal{P S H}\left(\mathbb{C}^{n}\right)$ such that $v^{-1}(-\infty) \cap \Omega=E$. Now the set $v^{-1}(-\infty)$ is complete pluripolar in $\mathbb{C}^{n}$, so by Theorem 2.5 we get $u \in \mathcal{L}\left(\mathbb{C}^{n}\right)$ so that $u^{-1}(-\infty)=v^{-1}(-\infty)$. We are done.

Let $E \subset \mathbb{C}^{n}$. Then the Siciak extremal function associated to $E$ is defined as follows:

$$
V_{E}(z)=\sup \left\{u(z): u \in \mathcal{L}\left(\mathbb{C}^{n}\right),\left.u\right|_{E} \leq 0\right\} .
$$

Siciak has proved in [Sic] that if $E$ is a pluripolar set in $\mathbb{C}^{n}$ then so is $A_{V_{E}}=\left\{z \in \mathbb{C}^{n}: V_{E}(z)<\infty\right\}$. The result below describes the pluripolar hull of $A_{V_{E}}$ and $A_{V_{E^{*}}}$.

Proposition 5.3. Let $E \subset \mathbb{C}^{n}$ be a pluripolar set. Then

$$
\left(A_{V_{E}}\right)^{*}=A_{V_{E^{*}}}=E^{*} \text {. }
$$

Consequently, if $E=E^{*}$ then $E^{*}=A_{V_{E}}=\left\{z \in \mathbb{C}^{n}: V_{E}(z)<\infty\right\}$.

Proof. First we prove that $A_{V_{E^{*}}}=E^{*}$. Since $V_{E^{*}} \equiv 0$ on $E^{*}$ we deduce that $E^{*} \subset A_{V_{E^{*}}}$. For the reverse inclusion, we fix $z \in A_{V_{E^{*}}}$ and let $u \in$ $\mathcal{L}\left(\mathbb{C}^{n}\right)$ with $u \equiv-\infty$ on $E$. From the definition of $E^{*}$ we have $u \equiv-\infty$ on $E^{*}$. Hence for every $m>0$ we have $u+m \in \mathcal{L}\left(\mathbb{C}^{n}\right)$ and $u+m \equiv-\infty$ on $E$. Consequently, $u(z)+m \leq V_{E^{*}}(z)$. This leads to $u(z) \leq V_{E^{*}}(z)-m$. 
Now let $m$ go to $\infty$ to obtain $u(z)=-\infty$. Applying Proposition 5.1 we get $z \in \widetilde{E}=E^{*}$. The proof is complete.

6. Miscellanea. To finish this paper, we include the following elementary facts.

Proposition 6.1. Let $E$ be a compact pluripolar subset of a pseudoconvex domain $\Omega$ in $\mathbb{C}^{n}$. Let $\widehat{E}_{\Omega}$ denote the holomorphic hull of $E$ in $\Omega$, i.e.,

$$
\widehat{E}_{\Omega}=\left\{z \in \Omega:|f(z)| \leq\|f\|_{E}, \forall f \text { holomorphic on } \Omega\right\} .
$$

Then $\widehat{E}_{\Omega} \subset E_{\Omega}^{*}$.

Proof. Let $u \in \mathcal{P} \mathcal{S H}(\Omega)$ be such that $u \equiv-\infty$ on $E$. Since $\Omega$ is pseudoconvex, it is well known that $\widehat{E}_{\Omega}$ coincides with the hull of $E$ with respect to plurisubharmonic functions, and we infer that $u \equiv-\infty$ on $\widehat{E}_{\Omega}$. The desired conclusion follows.

Proposition 6.2. Let $D, G$ be domains in $\mathbb{C}^{n}$ and $\mathbb{C}^{m}$ respectively and let $E \subset D, F \subset G$ be pluripolar sets. Then

$$
\begin{aligned}
& (E \times F)_{D \times G}^{*}=E_{D}^{*} \times F_{G}^{*}, \\
& (E \times F)_{D \times G}^{-}=E_{D}^{-} \times F_{G}^{-} .
\end{aligned}
$$

Proof. It is enough to prove (6), since the proof of the other equality is similar. For (6), we only have to show

$$
E_{D}^{*} \times F_{G}^{*} \subset(E \times F)_{D \times G}^{*}
$$

as the reverse inclusion is trivial. To this end, we claim that

$$
E_{D}^{*} \times F \subset(E \times F)_{D \times G}^{*} .
$$

Indeed, let $\left(z_{0}, w_{0}\right) \in E_{D}^{*} \times F$ and $\varphi(z, w) \in \mathcal{P S H}(D \times G)$ and $\left.\varphi\right|_{E \times F}=-\infty$. Then $\varphi\left(z, w_{0}\right) \in \mathcal{P S H}(D)$ and $\left.\varphi\right|_{E}=-\infty$. Hence, $\varphi\left(z_{0}, w_{0}\right)=-\infty$ and $\left(z_{0}, w_{0}\right) \in(E \times F)_{D \times G}^{*}$. Thus $(7)$ is proved. Replace $F$ in $(7)$ by $F_{G}^{*}$ to obtain

$$
E_{D}^{*} \times F_{G}^{*} \subset\left(E \times F_{G}^{*}\right)_{D \times G}^{*} .
$$

On the other hand, interchanging the roles of $E$ and $F$ in (7) gives

$$
E \times F_{G}^{*} \subset(E \times F)_{D \times G}^{*} .
$$

Combining (8) and (9) we get (6).

\section{References}

[BT] E. Bedford and A. Taylor, Plurisubharmonic functions with logarithmic singularities, Ann. Inst. Fourier (Grenoble) 38 (1988), 133-171. 
[Ch] E. Chirka, Expansion in series and the degree of rational approximation of holomorphic functions with analytic singularities, Math. USSR-Sb. 22 (1974), 323332.

[E] A. Edigarian, Pluripolar hulls and holomorphic coverings, Israel J. Math. 130 (2002), 77-92.

[EW] A. Edigarian and J. Wiegerinck, Determination of the pluripolar hull of graphs of certain holomorphic functions, preprint, 2003.

[FN] J. Fornæss and R. Narasimhan, The Levi problem on complex spaces with singularities, Math. Ann. 248 (1980), 47-72.

[Kl] M. Klimek, Pluripotential Theory, London Math. Soc. Monographs 6, Clarendon Press, 1991.

[La] N. S. Landkoff, Foundations of Modern Potential Theory, Grundlehren Math. Wiss. 180, Springer, Berlin, 1972.

[LP] N. Levenberg and E. A. Poletsky, Pluripolar hulls, Michigan Math. J. 46 (1999), 151-162.

[Ri] R. Richberg, Stetige streng pseudokonvexe Funktionen, Math. Ann. 175 (1968), 257-286.

[Sib] N. Sibony, Quelques problèmes de prolongement de courants en analyse complexe, Duke Math. J. 52 (1985), 157-197.

[Sic] J. Siciak, Extremal plurisubharmonic functions on $\mathbb{C}^{n}$, Ann. Polon. Math. 39 (1981), 175-211.

[Wi1] J. Wiegerinck, The pluripolar hull of $e^{-1 / z}$, Ark. Mat. 38 (2000), 201-208.

[Wi2] - Graphs of holomorphic functions with isolated singularities are complete pluripolar, Michigan Math. J. 47 (2000), 191-197.

[Wi3] —, Pluripolar sets: hulls and completeness, in: G. Raby \& F. Symesak (eds.), Actes des rencontres d'analyse complexe, Atlantique, 2002, 209-219.

[Ze] A. Zeriahi, Ensembles pluripolaires exceptionnels pour la croissance partielle des fonctions holomorphes, Ann. Polon. Math. 50 (1989), 81-91.

Department of Mathematics

University of Education

Tuliem, Hanoi, Vietnam

E-mail: mauhai@fpt.vn

ngquangdieu@hn.vnn.vn

Reçu par la Rédaction le 1.8.2004

Révisé le 18.12.2004 